\title{
Empirical Mode Decomposition Template Matched Filter for Detection and Estimation of T-Wave Alternans
}

\author{
Asim D. Bakhshi ${ }^{1}$,Muhammad Latif ${ }^{2}$, Sajid Bashir ${ }^{1}$, Hafiz M. Ali ${ }^{3}$ \\ ${ }^{1}$ Department of Electrical Engineering, Abasyn University, Islamabad Campus, Pakistan. \\ ${ }^{2}$ College of Electrical and Mechanical Engineering, National University of Sciences \& Technology, \\ Islamabad, Pakistan, \\ ${ }^{3}$ School of Engineering Science, Simon Fraser University, Burnaby BC, Canada
}

\begin{abstract}
T-wave alternans (TWA) is a well-recognized marker for malignant cardiac arrhythmia that may lead to sudden cardiac death. TWA detection and estimation becomes a challenge under the presence of process noises, transient outliers and physiological artifacts. We propose an empirical mode decomposition (EMD) based template matched filter approach for TWA analysis, targeting detection of distributed alternans energy rather than peak amplitude. EMD is performed to construct the template for matched filter which is central to alternans estimation and detection. The template is dynamically generated through decomposition into intrinsic mode functions (IMFs) and subsequent reconstruction. Notable improvement is achieved through correlation based reconstruction that shows a performance gain of $>2 d B$ over other approaches including the spectral method (SM).
\end{abstract}

\section{Introduction}

Cardiovascular issues are leading cause of mortality estimated to result about $30 \%$ or 17 million annual deaths worldwide. More importantly sudden cardiac death (SCD) triggers about $40-50 \%$ of the total cardiovascular fatalities [1]. Presence of T-wave alternans (TWA) also referred to as repolarization alternans is regarded as the prime reason of SCD. The alternan consists of a periodic beat-to-beat alternating change in the amplitude and / or shape of the ST-T complex as shown in figure 1 (adapted from [2]).

Despite extensive research in ECG denoising [3] TWA analysis remains challenging. The challenge stems from the presence of various type of process noise including electrode movement and muscular activity. Detection and estimation of TWA is adversely influenced by these anomalies and hence necessitate accurate statistical model incorporating physiological noises and other outliers [4]. The non-stationary nature of ECG and inter-beat variations make the scenario even more intricate. Reported TWA analysis algorithms are categorized in three broad classes as: the short-term Fourier transform (STFT) based techniques, methods based on counting of sign changes, and nonlinear filtering [5]. Illustrious algorithms include spectral method (SM) [6], modified moving average method (MMAM) [7], correlation method (CM) [8] and the multilead application based on Laplacian Likelihood Ratio Method (LLR) [2].

In this paper we incorporate EMD for construction of matched-filter template and extend the performance of the median matched filter (MMF) proposed by the authors in [9]. EMD is employed for separating useful information from noise and other artifacts through decomposition of measured signal into IMFs and subsequent reconstruction. Monte Carlo simulations are used to show the performance comparison of proposed EMD based template matched filter (EMF) with well known existing methods. Various reconstruction options are exercised that show significant performance improvement especially when correlation based method is adopted. It is also shown that this improved-EMF (I-EMF) attains a performance gain $>2 \mathrm{~dB}$ over all the above algorithms including SM. In Section 2 we discuss the materials and methods used, Section 3 shows results and discussions and Section 4 concludes the presented work.

\section{Materials and Methods}

\subsection{Pre-processing of ECG}

Before further analysis, pre-processing is done for conditioning ECG signal; QRS detection followed by segregation of ST-T complexes and alignment of segregated complexes. The output of the pre-processing stage can be represented as an $N \times M$ matrix of ST-T segments:

$$
\mathbf{X}=\left[\mathbf{x}_{0}, \ldots, \mathbf{x}_{M-1}\right]
$$




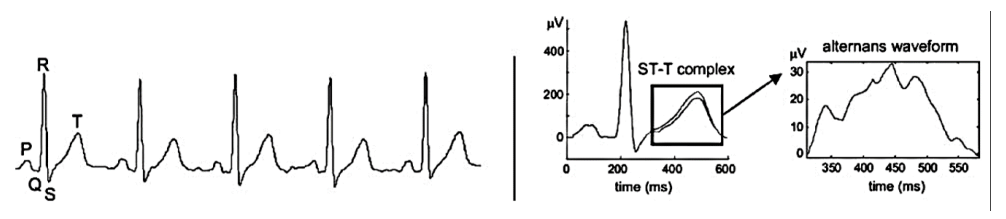

Figure 1. T-waves aligned in time and superimposed to highlight TWA phenomenon.

where $\mathbf{x}_{k}=\left[x_{k}[0], \ldots, x_{k}[N-1]\right] ; k=1, \ldots, M$ is the $k$ th ST-T complex and $N$ represents the number of data samples in each ST-T segment.

\subsection{System Model}

An $L$ beat TWA analysis window is selected from the pre-processed $M$ segmented ST-T complexes. The analysis window has following matrix notation:

$$
\mathbf{S}=\mathbf{X}+\mathbf{W}
$$

where $\mathbf{W} \in \mathbb{R}^{N \times L}$ represent samples of the Gaussian noise. If the even and odd ST-T complexes are denoted as $\mathbf{E} \in \mathbb{R}^{N \times L / 2}$ and $\mathbf{O} \in \mathbb{R}^{N \times L / 2}$ respectively, then (2) for $l$ th beat can be expressed as:

$$
\begin{aligned}
& \mathbf{e}_{i}=\mathbf{x}_{l}+\mathbf{w}_{l} \\
& \mathbf{o}_{i}=\mathbf{x}_{l+1}+\mathbf{w}_{l+1}
\end{aligned}
$$

where $i=1, \ldots, L / 2 ; \mathbf{x}_{l} \in \mathbb{R}^{N \times 1}$ and $\mathbf{w}_{l} \in \mathbb{R}^{N \times 1}$ are the $l$ th column of $\mathbf{X}$ and $\mathbf{W}$ respectively. Let the matrix $\mathbf{U}=\left[\begin{array}{lll}\mathbf{u}_{1} & \cdots & \mathbf{u}_{L / 2}\end{array}\right]$ represents the difference between consecutive ST-T complexes, then $\mathbf{u}_{i}=\mathbf{o}_{i}-\mathbf{e}_{i}$. TWA is then expressed as:

$$
\mathbf{g}_{i}=\mathbf{u}_{i}-\phi_{i}
$$

is a vector encompassing $\mathbf{u}_{i}$ and the residue noise $\phi_{i}=$ $\mathbf{w}_{l+1}-\mathbf{w}_{l}$ corresponding to the $i$ th pair of consecutive ST-T complexes.

\subsection{Matched Filter}

Matched filter is used for detection where a template of the required input exists or can be obtained / estimated. We make an effort of using matched filter for detection of TWA. The test statistic $z\left(\mathbf{u}_{i}\right)$ may be calculated as:

$$
z\left(\mathbf{u}_{i}\right)=\mathbf{u}_{i}^{T} \mathbf{g}_{i}
$$

where $(\cdot)^{T}$ represents the vector transpose operation. The finite impulse response implementation of (5) results in the classical matched filter that maximizes SNR at the output and gives optimal detection performance. The alternan waveform $\mathbf{g}_{i}$ is generally unknown (albeit deterministic), hence, the proposed detector is not classically optimal.
We propose to replace $\mathbf{g}_{i}$ with a known template $\hat{\mathbf{g}}_{i}$, making the detector as quasi optimal. Median and mean templates have been used by Sajid et al. suggesting new algorithms as mean and median matched filter based TWA detector [9]. We suggest obtaining the dynamic templates by getting the difference between a set of consecutive even and odd beats at first place i.e. $\mathbf{u}_{i}$ and then denoising it using EMD. We shall thus construct $L / 2$ templates from an observation window of length $L$.

\subsection{Empirical Mode Decomposition}

EMD is established upon data-driven mechanism that is most pertinent for analysis of natural signals characterized as non-linear, non-stationary and stochastic [10]. It is reported as an efficient denoising tool in various fields of signal processing $[11,12]$. EMD decomposes a signal into IMFs that satisfy [13]:

- Difference between number of extrema and zero crossings is zero or at maximum one.

- At any point the mean value (of envelopes defined by maxima and by minima of the function) is zero (or $\approx 0$ ).

EMD is applied to individual difference vector $\left(\mathbf{u}_{i}\right.$ in (4)) independently. Process yields a template as under:

1. Sifting Process

- Determine the local maxima vector and local minima vector of the difference vector $\mathbf{u}_{i}$.

- Apply cubic spline interpolation to the local maxima and minima vectors to build the upper $\mathbf{u}_{e}[n]$ and lower $\mathbf{l}_{e}[n]$ envelopes respectively. Find the mean envelope:

$$
\mathbf{m}[n]=\frac{\mathbf{u}_{e}[n]+\mathbf{l}_{e}[n]}{2}
$$

- Find corresponding proto IMF as $\mathbf{h}[n]=\mathbf{u}_{i}[n]-\mathbf{m}[n]$. 2. Stopping Criterion: Calculate sum of differences (SD) i.e. normalized squared difference between successive $\mathbf{h}[n]$ (step-1) and compare against a pre-defined threshold:

$$
\mathrm{SD}_{k}=\frac{\sum_{n=0}^{N-1}\left(h_{k-1}[n]-h_{k}[n]\right)^{2}}{\sum_{n=0}^{N} h_{k-1}^{2}[n]}
$$

where $h_{k}[n]$ is the $n$th sample of the $k$ th proto IMF. If proto IMF satisfies the stopping criterion it is declared as a legitimate IMF, $\mathbf{c}[n]$. 


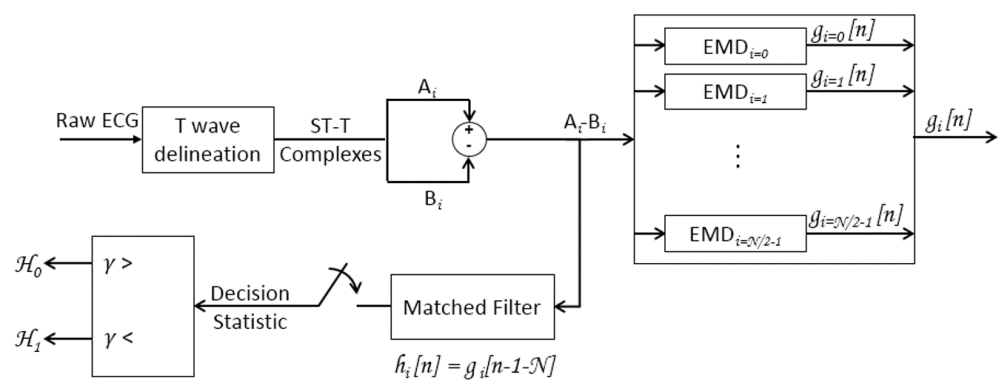

Figure 2. Schematic representation of TWA analysis using EMF.

3. Determine the residual vector

$$
\mathbf{r}[n]=\mathbf{u}_{i}[n]-\mathbf{c}[n]
$$

If $\mathbf{r}[n]$ is a constant or a monotonic function, terminate; otherwise declare it as the new data set and go to Step-1.

\section{5. $\quad$ EMD Based Matched Filter (EMF)}

EMD processing over a single difference vector $\mathbf{u}_{i}[n]$ yields $M$ IMFs and a residue signal such that:

$$
\mathbf{u}_{i}[n]=\sum_{j=\mathrm{o}}^{M-1} \mathbf{c}_{j}[n]+\mathbf{r}[n]
$$

We then apply Hjorth descriptors to individual IMFs and residue signal in order to calculate spectral purity index (SPI) for each [13]. Based upon SPI, we discard IMFs responsible for noise and reconstruct the noiseless template $\hat{\mathrm{g}}_{i}[n]$ by adding the remaining components. These templates are used for implementation of eq (5).

\section{Results and Discussions}

The simulation model shown in figure 2 is followed to validate the performance of the suggested algorithm. ST-T segments obtained from $M$ beats of synthetic ECG are segregated as even and odd. For each even-odd pair $\left(\mathrm{A}_{i}, \mathrm{~B}_{i}\right)$, the difference being the alternan waveform, is matched against a corresponding dynamically generated template through decomposition into IMFs and subsequent reconstruction. TWA of known magnitude is added in alternate beats of background signal arbitrarily obtained from ECG signals of MIT-BIH Arrhythmia Database [14]. In presence of additive Gaussian noise, comparison of proposed technique with other TWA detectors is carried out by performing Monte Carlo simulations.

Detection performance of different techniques with TWA magnitude fixed at $20 \mu \mathrm{V}$ is presented in figure 3 for an SNR range from -15 to $30 \mathrm{~dB}$. The proposed detector outperforms MMF over the entire range of SNR and its performance is comparable with SM and $\mathrm{CM}$ for $\mathrm{SNR} \geq$ $14 \mathrm{~dB}$.

The estimation accuracy of different algorithms is shown in shown in figure 4. It can be observed that EMF performs better than MMAM over the entire range of SNR and is better than CM for SNR of $25 \mathrm{~dB}$ and above.

In the simulation results of figure 3 an SPI based upon Hjorth descriptors is utilized for reconstruction of the template, as used by Velasco et al. [13]. This resulted in a detection performance by the proposed algorithm surpassing MMAM only. In order to further improve the results, we studied different variations of EMD and the improvements therein are referred to as improved empirical mode decomposition (I-EMD). Our focus was the template reconstruction phase after the EMD has been applied on the input signal. Following summarizes this effort:

- As first improvement, cross correlation between individual IMFs and the signal is calculated; IMFs which are more correlated with the original signal are summed together to form up the reconstructed signal.

- As second improvement, we calculated Hurst exponents of individual IMFs using detrended fluctuation analysis (DFA). IMFs with lesser value of Hurst exponents represent more randomness and are discarded because they constitute noise.

EMF with all three variants of EMD (original EMD and two I-EMDs) is pitched against the bench mark algorithm i.e. SM. The results are shown in figure 5. It can be observed that the proposed algorithm with template reconstruction using cross correlation criterion outperforms SM by $2 \mathrm{~dB}$ over entire working range of SNR.

\section{Conclusion}

In this study an EMD based TWA analysis algorithm has been proposed. EMD, in the introduced methodology EMF has been used for constructing dynamic templates. The background of the proposed methodology is derived from matched filter theory, where EMD based templates are used to match the alternan signal. The SNR, because of built in characteristic of matched filter, is maximized at the 


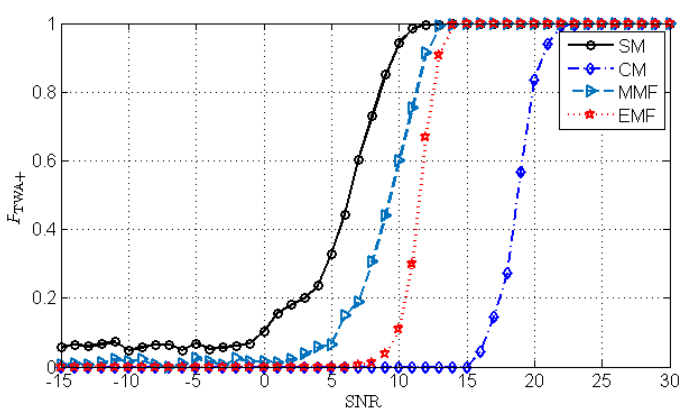

Figure 3. Detection results for various TWA algorithms with alternan amplitude of $20 \mu \mathrm{V}$.

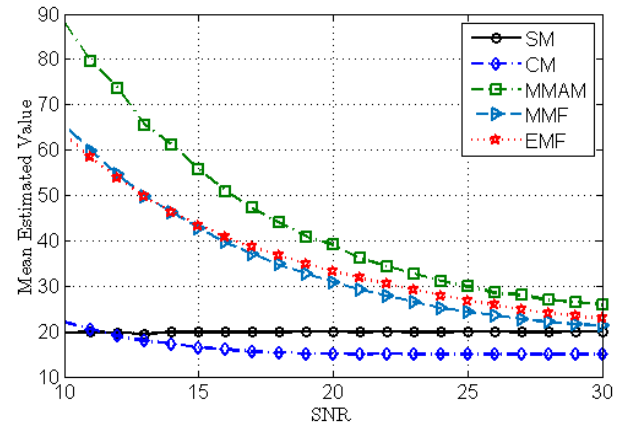

Figure 4. Mean estimated value versus SNR for various TWA algorithms for an alternan amplitude of $20 \mu \mathrm{V}$.

filter output; thus making easy the discrimination of TWA from noise. Improvements in reconstruction phase after EMD implementation, have also been considered. These improvements i.e. I-EMDs include use of Hurst exponents and cross correlation instead of Hjorth descriptors for reconstructing the templates. Performance of EMF (both EMD and I-EMD based) has been compared with the state of the art TWA analysis algorithms. I-EMD based upon cross correlation has further improved detection performance. Its matched filter implementation has surpassed the bench mark SM over entire working range of SNR.

\section{References}

[1] Mehra R. Global public health problem of sudden cardiac death. Journal of electrocardiology 2007; 40 (6 Suppl): 118-22.

[2] Monasterio V, Laguna P, and Martı́nez JP. Multilead analysis of T-wave alternans in the ECG using principal component analysis. IEEE Trans in Biomed Eng, 2009; 57 (7): 1880-90.

[3] Martinez JP and Olmos S. Methodological principles of T wave alternans analysis: a unified framework. IEEE Trans on Biomed Eng 2005; 52 (4): 599-613.

[4] Bakhshi AD and Bashir S and Maud MA. An improved statistical representation for ECG electrode movement and

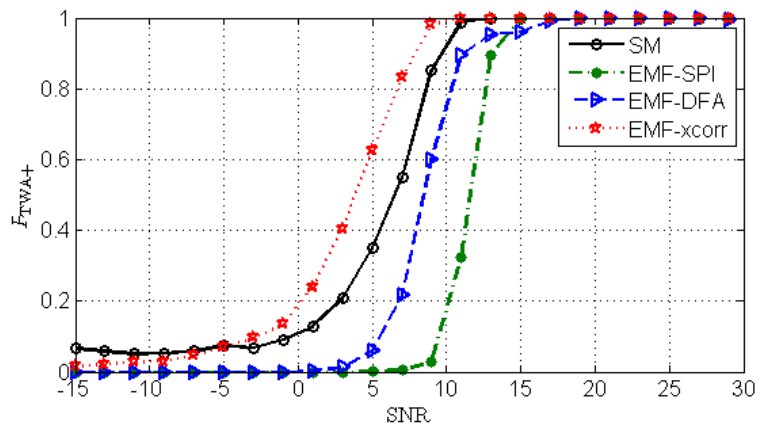

Figure 5. Detection results achieved by I-EMDs based algorithm for an alternan amplitude of $20 \mu \mathrm{V}$.

muscular activity noises in the context of T-wave alternan estimation. Biomed Sig Processing and Con 2013; 8(3): 297-301.

[5] Laguna P and Martı́nez, JP and Pueyo E. Techniques for ventricular repolarization instability assessment from the ECG. Proceedings of the IEEE 2016; 104 (2): 392-415.

[6] Rosenbaum DS, Jackson JE, Smith JM, Garan, J. N H. Ruskin, and Cohen RJ. Electrical alternans and vulnerability to ventricular arrhythmias. New England Journal of Medicine 1994; 330 (4): 235-41.

[7] Nearing BD and Verrier VL. Modified moving average analysis of t-wave alternans to predict ventricular fibrillation with high accuracy. Journal of Applied Physiology 2002; 92(2): 541-9.

[8] Burattini WZ, and Moss AJ. Correlation method for detection of transient t-wave alternans in digital holter ecg recordings. Annals of noninvasive Electrocardiology 1999; 4 (4); 416-24.

[9] Bashir S, Bakhshi AD and Maud MA. A template matchedfilter based scheme for detection and estimation of $t$-wave alternans. Biomed Sig Processing and Con 2014; 13: 247 61.

[10] Weng B and Blanco-Velasco M and Barner KE. ECG denoising based on the empirical mode decomposition. Eng in Medicine and Biology Society, 2006 : 1-4.

[11] Salman AH and Ahmadi N, Mengko R and Langi Armein ZR and Mengko Tati LR. Empirical Mode Decomposition Based Denoising Method for Heart Sound Signal and Its Performance Analysis. International Journal of Electrical and Computer Eng (IJECE) 2016: 6(5).

[12] Haritha $C$ and Ganesan, $M$ and Sumesh, EP. A survey on modern trends in ECG noise removal techniques. International Conference on Circuit, Power and Computing Tech (ICCPCT) 2016: 1-7.

[13] Blanco-Velasco M, Cruz-Roldan F and Godino-Liorente, JI and Barner KE. Nonlinear trend estimation of the ventricular repolarization segment for T-wave alternans detection. IEEE Trans on Biomed Eng 2010; 57 (10): 2402-12.

[14] Goldberger AL et al. PhysioBank, PhysioToolkit, and PhysioNet: Components of a New Research Resource for Complex Physiologic Signals. Circulation 2000; 101 (23): e215e220. 